\title{
Understanding the linkage between different measure of Obesity/overweight and Pre- diabetes/diabetes Prevalence among Adolescents (10-19 years) in India: A Gendered perspective
}

\section{Pradeep kumar}

International Institute for Population Sciences

Shobhit Srivastava

International Institute for Population Sciences

Prem Shankar Mishra ( $\sim$ psmishrabhu@gmail.com)

Institute for Social and Economic Change

Research article

Keywords: Pre-diabetes/diabetes, obesity, adolescents, India

Posted Date: October 7th, 2020

DOI: https://doi.org/10.21203/rs.3.rs-47939/v1

License: (c) (i) This work is licensed under a Creative Commons Attribution 4.0 International License.

Read Full License

Version of Record: A version of this preprint was published at BMC Endocrine Disorders on July 7th, 2021. See the published version at https://doi.org/10.1186/s12902-021-00802-w. 


\section{Abstract \\ Background}

The International Diabetes Federation (IDF) estimated that 1.1 million children and adolescents aged 1419 years are living with diabetes. Diabetes is a chronic, progressive disease characterized by elevated levels of blood glucose. It is also recognized as a complex disease that affects people of different ages due to different causes. The present study aims to estimate the prevalence of pre-diabetes/diabetes at the national level. Additionally, the respective study determines the factors associated with prediabetes/diabetes conditions among adolescents at the national level.

\section{Methods}

The data for this study was carried out from Comprehensive National Nutrition Survey (CNNS), the firstever nationally representative nutrition survey of children and adolescents in India. The study used a sample size of 17,865 male and 17,965 female adolescents for the analysis. Descriptive statistics, bivariate analysis, and logistic regression analysis were done to carve out the results.

\section{Results}

The prevalence of pre-diabetes/diabetes was $12.3 \%$ and $8.4 \%$ among male and female adolescents in India respectively. Body mass index and Subscapular skinfold thickness were the two most important predictors of pre-diabetes/diabetes among adolescents. Further, physical activities show a negative association with pre-diabetes/diabetes. Moreover, interaction models in the present study clearly reveal the fact that females were less likely to suffer from pre-diabetes/diabetes than males. Additionally, it was found that the prevalence of pre-diabetes/diabetes was high among female adolescents from lower socio-economic strata.

\section{Conclusion}

Pre-diabetes/diabetes conditions can be prevented among adolescents with effective approaches available. Regular exercise and involvement in moderate to high physical activity, maintaining a healthy diet, avoiding substance use, and cholesterol levels are recommended to control pre-diabetes/diabetes conditions among adolescents.

\section{Background}

The International Diabetes Federation (IDF) estimated that 1.1 million children and adolescents aged 1419 years were living with diabetes [1]. In addition, increasing prevalence of pre-diabetes and diabetes led by obesity among the young population fuelled metabolic syndrome and obesity-related co-morbidities 
across the low and middle-income countries [2]. There is a global effort to curb down the rise of diabetes and obesity by 2025; however, a steady rise in the prevalence of diabetes is a matter of concern [3].

India alone contributes a larger proportion to premature deaths from non-communicable diseases (NCDs), including diabetes across all socio-economic groups [4]. Achieving the global agenda of Sustainable Development Goals (SDGs) by 2030 , by reducing one-third of premature mortality from NCDs-including diabetes, a universal healthcare access approach is therefore required in most developing countries, including India (3-4). India is currently going through the nutritional transition and it is evident with a rise in the prevalence of overweight and obesity [6]. Also, estimation shows that nearly 166 million adults are overweight/obese in 2016 in India [3]. In the case of adults who are affected by diabetes, the numbers are estimated to be nearly 73 million, the second-largest in the world [1].

Diabetes is a chronic, progressive disease characterized by elevated levels of blood glucose levels. It is also recognized as a complex disease that affects people of different agesdue to different causes. There are three main types of diabetes, type-1 diabetes (T1D), type-2 diabetes (T2D), and gestational diabetes mellitus (GDM)[3]. The type-1 diabetes incidence rate is increasing among the younger population and the contributing factors have remained unclear. Though there are factors that affect the heterogeneity of the respective disease such as genetic factors, behavioural risk factors, food and dietary habits, physical inactivity, lifestyle changes.

Furthermore, the prevalence of pre-diabetes/diabetes among children, adolescents, and younger adults is high and becomes severe due to rising levels of obesity [7]-[9]. physical inactivity [10], [11] and poor dietary habits [12]. which includes increased levels of substance use [13], [14] among adolescent groups. The spread of pre-diabetes/diabetes among the population is witnessed in light of socio-economic groups, rural-urban, sex, and family history of diabetes [15]-[17] Lack of physical activities and having a sedentary lifestyle among adolescents made responsible for cardiovascular disease and obesity-related risk factors which in turn may cause menace of pre-diabetes/diabetes among them [11], [18], [19]. Moreover, Triglycerides, Cholesterol, High-density lipoprotein (HDL), and Low-density lipoprotein (LDL) levels were found to be significant factors for the prevalence of pre-diabetes/diabetes among adolescents [20], [21].

The present study utilized the different forms of obesity which may affect the chaos of prediabetes/diabetes differently. Previous literature measured the five types of obesity in their study. For instance, obesity measured through body mass index [21], waist circumference [21], waist to hip ratio [22], Subscapular skinfold thickness (SSFT) [23] and Triceps skinfold thickness (TSFT) [23].

There exists the dearth of literature focusing on the association of pre-diabetes/diabetes with obesity levels among adolescents at the national level in India. Therefore, the present study aims to estimate the prevalence of pre-diabetes/diabetes at the national level. Additionally, the respective study determines the factors for pre-diabetes/diabetes conditions among adolescents at the national level. The study hypothesized that there was no association between pre-diabetes/diabetes conditions with obesity levels and physical activity among adolescents in India. 


\section{Methods}

The data for this study was carried out from Comprehensive National Nutrition Survey (CNNS), the firstever nationally representative nutrition survey of children and adolescents in India. The survey collects the data on the status of pre-school ( $0-4$ years), school-age children (5-9 years), and adolescents (1019 years) through interviews, a comprehensive set of anthropometric measures and biochemical indicators. The CNNS covered all 30 states of India using a multi-stage survey design for the selection of households and individuals aged 0-19 years. The data has been collected with the written consent of the participants. The survey was implemented under the guidance of the MoHFW, UNICEF, a Technical Advisory Group (TAG), and the Center for Disease Control (CDC). Data can be obtained on request from Population Council (India office) website. The detailed methodology, sampling design, and data collection procedure were published in the survey report [24]. The CNNS collected data from a total of 38,060 children aged $0-4$ years, 38,355 children aged $5-9$ years, and 35,830 adolescents age 10-19 years. The study used a sample size of 17,865 male and 17965 female adolescents for the analysis.

\section{Outcome variable}

The outcome variable was pre-diabetes/diabetes conditions among adolescents aged 10-19 years. The pre-diabetes/diabetes condition was measure through glycosylated haemoglobin (HbA1c) [25]. The cutoff points for pre-diabetes/diabetes among adolescents were as: pre-diabetes $(>5.6 \%$ and $\leq 6.4 \%)$ and diabetes $(>6.4 \%)$ [26]. We merged diabetes and pre-diabetes into one category due to the small sample in the diabetes condition. The outcome variable was divided into two categories as 0 "no prediabetes/diabetes" and 1 "pre-diabetes/diabetes".

\section{Exposure variables}

Body mass index was categorized as not overweight/obese (BMI $\leq+1 \mathrm{SD})$ and overweight/obese (BMI > $+1 \mathrm{SD}$ ) group, abdominal obesity was categorized as no (waist circumference-for-age $\leq+1 \mathrm{SD}$ ) and yes (waist circumference-for-age $>+1$ SD), Triceps skinfold thickness (TSFT) was grouped as not overweight (TSFT-for-age $\leq+1 \mathrm{SD}$ ) and overweight (TSFT-for-age $>+1 \mathrm{SD}$ ) and Subscapular skinfold thickness (SSFT) was divided into two categories: not overweight (SSFT-for-age $\leq+1 \mathrm{SD}$ ) and overweight (SSFTfor-age $>+1 \mathrm{SD}$ ). Physical activity was categorized as a low, medium, and high using principal component analysis approach as 15 questions were used to generate the respective variable. Serum triglycerides (assessed by spectrophotometry and enzymatic endpoint method) was categorized into low $(<130 \mathrm{mg} / \mathrm{dl}$ ) and high ( $\geq 130 \mathrm{mg} / \mathrm{dl})$, cholesterol (assessed by spectrophotometry using cholesterol oxidase esterase peroxidase) was recoded as low ( $<200 \mathrm{mg} / \mathrm{dl}$ ) and high ( $\geq 200 \mathrm{mg} / \mathrm{dl})$, low-density lipoprotein (LDL) (assessed by spectrophotometry and direct measure cholesterol oxidase) was categorized as low $(<130 \mathrm{mg} / \mathrm{dl})$ and high $(\geq 130 \mathrm{mg} / \mathrm{dl})$ and high-density lipoprotein (HDL) cholesterol (assessed by spectrophotometry and direct measure polyethylene glycol-modified cholesterol oxidase) was recoded as low ( $<40 \mathrm{mg} / \mathrm{dl}$ ) and high ( $\geq 40 \mathrm{mg} / \mathrm{dl}$ ). Age was categorized into early adolescents (1014 years) and late adolescents (15-19 years), education was divided into no schooling, dropouts and currently studying, Media exposure was categorized as no "no exposure to television \& radio \& newspaper 
\& internet" and yes "exposure to television or radio or newspaper or internet), parent education was recoded as (both uneducated, anyone educated and both educated), parent diabetes status was categorized as (no or anyone had), the diet was categorized as (vegetarian and non-vegetarian), Junk food consumption was categorized as (no, 1-2 days and 3-7 days), substance use was categorized as (no and yes). Caste was categorized as (Scheduled tribe/Scheduled caste (SC/ST) and non- Scheduled tribe/Scheduled caste), religion was categorized as (Hindu, Muslim, Christian, and Others), wealth quintile was categorized as (poor, middle and rich), the residence was categorized as (urban and rural) and region were divided as north, central, east, north-east, west, and south.

\section{Statistical analysis}

Descriptive analysis is used to show the sample profile of the respondents. Further, bivariate analysis was carried out to estimate the prevalence of pre-diabetes/diabetes by selected variables, and a proportion test was applied to test the significant difference between male and female adolescents for prediabetes/diabetes. Finally, multivariate logistic regression was performed to find out the effects of different predictors on pre-diabetes/diabetes. The results are presented in the form of an odds ratio (OR) with a $95 \%$ confidence interval $(\mathrm{Cl})$. The model is usually put into a more compact form as follows:

$$
\ln \left(\frac{P_{i}}{1-P_{i}}\right)=\beta_{0}+\beta_{1} x_{1}+\cdots+\beta_{M} x_{m-1}
$$

Where $\beta_{0}, \ldots . ., \beta_{M}$ are the regression coefficient indicating the relative effect of a particular explanatory variable on the outcome, the study further examined the possible interaction between body mass index, abdominal obesity, triceps skinfold thickness (TSFT), subscapular skinfold thickness (SSFT), and gender in models $2,3,4$, and 5 respectively.

\section{Results}

Anthropometric and socio-demographic profiles of adolescents aged 10-19 years are presented in Table 1. Overall, around five percent of male and female adolescents were overweight/obese and about two percent and one percent male and female adolescents have abdominal obesity. Nearly, five percent male and two percent female adolescents had triceps skinfold thickness whereas seven percent male and four percent female adolescents had subscapular skinfold thickness. About 14 percent male and 18 percent of female adolescents had high serum triglycerides. Moreover, almost the same proportion of adolescents (3\% vs. $5 \%$ ) had high cholesterol and low-density lipoprotein respectively. The proportion of adolescents was higher in the 10-14 years age group irrespective of gender. The majority of adolescents are currently studying and, around $17 \%$ male and $21 \%$ of female adolescents were school dropouts. More than three-fourth of adolescents had some mass media exposure. One-fourth of the parents had no schooling and a very low proportion of parents (anyone) had diabetes (4\%). About 81 percent male and $73 \%$ of female adolescents were non-vegetarian. Seven out of ten adolescents have consumed 1-2 days of junk food. The substance use was higher among male (8\%) adolescents than female ones. One-third 
of adolescents belonged to SC/ST group and the majority of adolescents were Hindu. Two-fifth of adolescents belonged to rich families and one-fourth of adolescents lived in urban areas.

Gender differentials for pre-diabetes/diabetes among adolescents presented in Fig. 1. Prediabetes/diabetes was more prevalent among 15 years of adolescent and it is lower in 10 years of the adolescent. Though, the prevalence of pre-diabetes/diabetes was higher among male adolescents compared to females irrespective of their age.

Table 2shows the gender differences in the prevalence of pre-diabetes/diabetes among adolescents aged 10-19 years across different socio-demographic characteristics in India. The prevalence of prediabetes/diabetes was higher among male adolescents across all the selected socio-demographic characteristics and differences were statistically significant. Substantial differences are observed among adolescents those who were having abdominal obesity (11.7\%), overweight (SSFT) (9.8\%), belonged to Northeast region (10.1\%), consumed junk food for 3-7 days ( $8.5 \%)$, and belonged to the middle $(6.4 \%)$ and rich families (5.6\%).

Binary logistic regression estimated for pre-diabetes/diabetes among adolescents aged 10-19 years were presented in Table 3. Results from model- 1 found that overweight/obese adolescents had $41 \%$ higher chances [Odd ratio (OR), $1.41 ; \mathrm{Cl}: 1.08-1.82$ ] to get pre-diabetes/diabetes compared to not overweight/obese adolescents. Moreover, SSFT has a significant effect on pre-diabetes/diabetes among adolescents. Overweight adolescents (SSFT) were significantly more likely (OR, 1.49: Cl: 1.15-1.94) to suffer from pre-diabetes/diabetes than those who were not overweight. There was a negative relationship between the physical activity of adolescents and their pre-diabetes/diabetes condition. Adolescents involved in high physical activity were less likely to suffer from pre-diabetes/diabetes compared to those who did low physical activities. Though the result was not significant for physical activities.

Model 2 represents the interaction between body mass index and gender for pre-diabetes/diabetes. Interestingly, a female adolescent with not overweight/obese category were significantly less likely to suffer (OR, 0.72; $\mathrm{Cl}: 0.62-0.83)$ from pre-diabetes/diabetes compared to male adolescent belonged to not overweight/obese category. Interaction between gender and abdominal obesity for pre-diabetes/diabetes condition were presented in Model 3. Male adolescents having abdominal obesity were more likely (OR, 1.26; Cl: $0.74-2.17$ ) to suffer from pre-diabetes/diabetes than a male adolescent with no abdominal obesity. Moreover, a female adolescent with abdominal obesity were significantly less likely (OR, 0.73 ; $\mathrm{Cl}$ : 0.63-0.84) to suffer from pre-diabetes/diabetes compared to a male adolescent with no abdominal obesity. Results from Model 4 revealed the interaction between gender and triceps skinfold thickness (TSFT) for pre-diabetes/diabetes among adolescents. The likelihood of pre-diabetes/diabetes among overweight (TSFT) male adolescent were lower (OR, 0.96; Cl: 0.63-1.45) than a not overweight male adolescent. However, the chances of pre-diabetes/diabetes among females with not overweight category were significantly lower (OR, 0.72; Cl: 0.62-0.83) compared to males with no overweight category. Model 5 shows the interaction between gender and subscapular skinfold thickness (SSFT) for prediabetes/diabetes among adolescents. SSFT has a significant effect on diabetes among both male and 
female adolescents. The probability of getting diabetes was significantly higher (OR, 2.23; Cl: 1.53-3.23) among overweight (SSFT) male adolescents than not overweight males. Moreover, a female adolescent from the not overweight category was significantly less likely (OR, 0.72 ; $\mathrm{Cl}$ : $0.62-0.84)$ to suffer from prediabetes/diabetes compared to males from not overweight category.

\section{Discussion}

The overall prevalence of pre-diabetes/diabetes was $12.3 \%$ and $8.4 \%$ among male and female adolescents in India respectively. Moreover, it was found that body mass index (BMI) and subscapular skinfold thickness (SSFT) was significantly associated with pre-diabetes/diabetes among adolescents in India. Additionally, it was revealed that female adolescents were less likely to suffer from prediabetes/diabetes than male adolescents in every category of obesity measure.

Earlier studies found that no accessibility of proper care such as early diagnosis, diabetes care management, and blood glucose testing available in primary healthcare settings in the early phases of life for children and adolescents [15], [27]. At this moment, it needs to be diagnosed (adolescent's diabetes or pre-diabetes) that can have a lesser risk in the longer term than the person who lives with undiagnosed and untreated diabetes.

The current study reveals the fact that there was a positive association between BMI, SSFT, and low physical activity status with pre-diabetes/diabetes conditions among adolescents in India. The results were consistent with previous findings that increased BMI was positively associated with prediabetes/diabetes conditions among adolescents [19]. Moreover, SSFT was also a significant risk factor of pre-diabetes/diabetes conditions among adolescents as argued by previous literature [28]-[30]. Apart from obesity, genetic and metabolic factors there are other significant risk factors but in the present study, those were not taken into account due to data limitations [2], [27], [31]. Additionally, low physical activity status was found to be a substantial risk factor for pre-diabetes/diabetes conditions among adolescents as found in previous studies [32]. It was too argued that even a moderate level of physical activity could help in the prevention of pre-diabetes conditions by reducing obesity levels among adolescents [32].

Dietary practices with unhealthy food such as high intake of junk foods and sugar-sweetened beverages are associated with a high prevalence of obesity/overweight that may lead to pre-diabetes/diabetes conditions among adolescents [33], [34]. This finding is consistent with findings of the present that adolescents with junk food consumption frequency of 3-7 days a week had a high prevalence of diabetes/diabetes condition. Additionally, the menace of substance use among adolescents was a staunch factor for pre-diabetes/diabetes conditions. The finding was paralleled with the previous study that abnormal alcohol and drug use among adolescents increases the risk of pre-diabetes/diabetes conditions [35].

Although it was earlier argued that diabetes is an affluent disease and it occurs among the population from higher socio-economic strata [15], however, the present study revealed that the disease pattern is now shifting towards lower affluent population, for instance, per-diabetes/diabetes condition was more 
among SC/ST, poor and adolescents from rural areas especially in case of females. Further research is needed to look into the issue.

The results showed that pre-diabetes/diabetes vary across gender for instance gender differential was over $4 \%$ i.e., males are more prone to pre-diabetes/diabetes than females. Moreover, for most of the background factors males were at high risk than females. Even previous literature argued that females do have a lower probability to suffer from pre-diabetes/diabetes [36]. The plausible reasons can be derived through descriptive statistics in the present study where it was clearly visible that obesity levels and substance use were low among females and which may further protect them from the burden of prediabetes and diabetes condition in comparison to their male counterpart. Additionally, interaction models revealed that females were less likely to be pre-diabetic/diabetic in comparison to males for the obesity measure. The findings are found to be consistent with the previous one as it was argued that female adolescents are less likely to be overweight or obese [37].

\section{Limitation}

The study has potential limitations. This study used cross-sectional data that does not allow to establish a causal relationship between variables. Despite the limitation, the study also has its own strength. This study used large scale survey data of adolescents which is the first-ever survey on nutrition and gives reliable estimates on diabetes at the national level.

\section{Conclusion}

Our study found that pre-diabetes/diabetes was highly prevalent among adolescents especially among males in India. Interaction models in the present study clearly reveal the fact that females are less likely to suffer from pre-diabetes/diabetes than males. BMI and SSFT are the two most important predictors of pre-diabetes/diabetes among adolescents in India. The high prevalence of pre-diabetes/diabetes among female adolescents from lower socio-economic strata poses a high concern in the Indian scenario. The emerging type 1 diabetes (T1D) among children and adolescents cannot be prevented with existing knowledge [3]. However, type 2 diabetes can be prevented with effective approaches available, and premature deaths can be curtailed off [3]. To avoid diabetes and related risk factors, regular exercise, maintaining a healthy diet, avoiding substance use, and controlling blood pressure and lipids as recommended by WHO guidelines [3]. The present study also suggests that to prevent prediabetes/diabetes conditions among adolescents certain health interventions are required. Utilization of knowledge, awareness, and programme (KAP) re-intervention to control obese/overweight among adolescents on the one hand and the regular health check-ups among adolescents on the other hand will surely help to curtail pre-diabetes/diabetes conditions among adolescents in India. Adolescent subpopulations are out of the coverage of the special programme that addresses the crucial public health issues of diabetes and related various risk factors.

\section{Abbreviations}


MoHFW: Ministry of Health and Family Welfare, UNICEF:United Nations Children Funds CNNS:Comprehensive National Nutrition Survey, BMI:Body Mass Index, HbA1c:glycosylated haemoglobin,T1D:Type-1 diabetes, T2D:Type-2 diabetes,GDM:gestational diabetes mellitus, TSFT:Triceps skinfold thickness, SSFT:Subscapular skinfold thickness,LDL:low-density lipoprotein, HDL:high-density lipoprotein,SC/ST:Scheduled Tribe/Scheduled Caste

\section{Declarations}

\section{Ethics approval and consent to participate}

The study is based on secondary data which is publically available on request from cnns.pc@gmail.com. Therefore ethical approval and consent to participate is not required.

\section{Consent for publication}

Not applicable

\section{Availability of data and materials}

We have provided details of the data in the methodology section. The CNNS data can be obtained on request from the Population Council, Delhi (India). The report and the survey tools are also available on the website:https://www.popcouncil.org/uploads/pdfs/2019RH_CNNSreport.pdf and cnns.pc@gmail.com respectively.

\section{Competing Interests}

The authors declare that they have no competing interests.

\section{Funding}

Authors did not receive any funding to carry out this research.

\section{Author's Contribution}

The concept was drafted by SS; PK and SS contributed to the analysis design; PSM and SS advised on the paper and assisted in paper conceptualization; PSM, PK, and SS contributed to the comprehensive writing of the article. All authors read and approved the final manuscript.

\section{Acknowledgments}

The authors are thankful to Population Council (India) for providing the data for this study. The authors are also thankful to Aditya and Megha Mittal for providing financial support to carry out the CNNS. Their generous support enabled us to utilize the highest quality and innovative methods for conducting the world's largest comprehensive nutrition survey. 


\section{References}

1. IDF. IDF DIABETES ATLAS Eighth edition 2017. 2017.

2. Tester J, Sharma S, Bradner C, Mietus-snyder M, Tinajero-deck L. Diabetes \& Metabolic Syndrome: Clinical Research \& Reviews Gender differences in prediabetes and insulin resistance among 1356 obese children in Northern California. Diabetes Metab Syndr Clin Res Rev. 2013;7(3):161-5.

3. WHO. "Consideration of the evidence on childhood obesity for the Commission on Ending Childhood Obesity Report of the Ad hoc Working Group on Science and Evidence for Ending Childhood Obesity," World Heal. Organ. Geneva, Switz., pp. 1-218, 2016.

4. Dandona L. and India State-Level Disease Burden Initiative Collaborators (167 authors), "Nations within a nation: variations in epidemiological transition across the states of India, 1990-2016 in the Global Burden of Disease Study," Lancet, vol. 390, pp. 2437-2460, 2017.

5. Psaltopoulou T, Tzanninis S, Ntanasis I, George S, Prevention and treatment of childhood and adolescent obesity: a systematic review of meta - analyses, no. 0123456789. Springer Singapore, 2019.

6. IIPS and ICF. "National Family Health Survey (NFHS-4), 2015-16," Mumbai, India, 2017.

7. Chatterjee S, Khunti K, Davies MJ. Type 2 diabetes. Lancet. 2017;6736(17):1-13.

8. Ramachandran A, et al. High prevalence of diabetes and impaired glucose tolerance in India: National Urban Diabetes Survey. Diabetologia. 2001;no. 44:1094-101.

9. Hills PAP, et al. Series Type 2 diabetes in south Asia 1 Epidemiology and determinants of type 2 diabetes in. LANCET Diabetes Endocrinol. 2018;8587(18):1-13.

10. Hills AP, Andersen LB, Byrne NM. Physical activity and obesity in children. Br J Sport Med. 2011;no. 45:866-70.

11. Kumar B, R. \& Robinson, Till S. "Physical activity and health in adolescence," vol. 15, no. 3, pp. 267272, 2015.

12. Lahiri A, Chakraborty A, Dasgupta U, Kumar A, Roy S, Bhattacharyya K. "Effect of Dietary Habit and Physical Activity on Overnutrition of Schoolgoing Adolescents: A Longitudinal Assessment in a Rural Block of West Bengal," pp. 171-177, 2019.

13. Ramachandran A. Snehalatha C. Mary S. Mukesh B. Bhaskar AD, Vijay V. The Indian Diabetes Prevention Programme shows that lifestyle modification and metformin prevent type 2 diabetes in Asian Indian subjects with impaired glucose tolerance (IDPP-1). Diabetologia. 2006;49:289-97.

14. NIGER DELTA Med. J., no. January, 2020.

15. Anjana RM, et al., "Articles Prevalence of diabetes and prediabetes in 15 states of India: results from the ICMR - INDIAB population-based cross-sectional study," vol. 8587, no. 17, 2017.

16. Gupta N, Shah P, Nayyar S, Misra A. "Childhood Obesity and the Metabolic Syndrome in Developing Countries," 2013. 
17. Patterson CC, Dahlquist GG, Gyürüs E, Green A, Soltész G, Group S. Incidence trends for childhood type 1 diabetes in Europe during 1989-2003 and predicted new cases 2005-20: a multicentre prospective registration study. Lancet. 2009;373(9680):2027-33.

18. Srinivasan BNA, Sudha M, Linda PS. Current Perspectives on Physical Activity and Exercise Recommendations for Children Spectrum Disorders. Am Phys Ther Assoc. 2014;94(6):875-89.

19. Breno Quintella Farah AHG-S, Aluısio Andrade-Lima, Christofaro RM, Diego Giulliano Destro, Mauro Virgı́lio Gomes de Barros, Wagner Luiz do Prado, Ritti-Dias, "Physical Activity and Heart Rate Variability in Adolescents with Abdominal Obesity," Pediatr Cardiol, vol. 39, pp. 466-472, 2018.

20. Khaodhiar L, Cummings S, Apovian CM. "Treating diabetes and prediabetes by focusing on obesity management," Current Diabetes Reports. 2009.

21. Al Amiri E, et al., "The prevalence, risk factors, and screening measure for prediabetes and diabetes among Emirati overweight/obese children and adolescents," BMC Public Health, 2015.

22. Alam DS, et al., "Overweight and abdominal obesity as determinants of undiagnosed diabetes and pre-diabetes in Bangladesh," BMC Obes., 2016.

23. Ganvir RH, Bhalla AK, Dayal D. "Growth Attainments of Indian Children with Type 1 Diabetes: A Mixed Longitudinal Study," Indian J. Pediatr., 2015.

24. Ministry of Health and Family. Welfare UNICEF, Council P. "Comprehensive National Nutrition Survey," 2019.

25. WHO. "Use of Glycated Haemoglobin (HbA1c) in the Diagnosis of Diabetes Mellitus Abbreviated Report of a WHO Consultation," 2011.

26. International Diabetes Federation. Globall IDF/IPAD Guideline for Diabetes in childhood and adolescence. 2011.

27. Mohan B, et al., "Prevalence of sustained hypertension and obesity among urban and rural adolescents: a school-based, cross- sectional study in North India," pp. 1-9, 2019.

28. Kim HM, Park J, Kim H, Kim HD. Prevalence of the metabolic syndrome in Korean adolescents aged 12-19 years from the Korean National Health and Nutrition Examination Survey 1998 and 2001. Diabetes Res Clin Pract. 2007;75:111-4.

29. Lambert M, Paradis G, Loughlin JO, Delvin EE, Hanley JA, Levy E, “PEDIATRIC HIGHLIGHT Insulin resistance syndrome in a representative sample of children and adolescents from Quebec, Canada," no. August, 2004.

30. Cruz ML, et al. The Metabolic Syndrome in Overweight Hispanic Youth and the Role of Insulin Sensitivity. J Clin Endocrinol Metab. 2004;89(1):108-13.

31. VS at al. Anjana, Pradeepa RM,R, Deepa M, Datta M. Prevalence of diabetes and prediabetes (impaired fasting glucose and / or impaired glucose tolerance) in urban and rural India: Phase I results of the Indian Council of Medical Research - INdia DIABetes (ICMR - INDIAB ) study. Diabetologia. 2011;no. 54:3022-7. 
32. Thomas AS, Greene LF, Ard JD, Oster RA, Darnell BE, Gower BA. "Physical activity may facilitate Diabetes prevention in Adolescents," Diabetes Care, 2009.

33. Lee YS, Med MP, Uk M. "Consequences of Childhood Obesity," vol. 38, no. 1, pp. 75-81, 2009.

34. Daniels SR. "Complications of obesity in children and adolescents," pp. 60-65, 2009.

35. Gold MA, Gladstein J. "Substance use among adolescents with diabetes mellitus: Preliminary findings," J. Adolesc. Heal., 1993.

36. Zamora-Kapoor A, Fyfe-Johnson A, Omidpanah A, Buchwald D, Sinclair K, "Risk factors for prediabetes and diabetes in adolescence and their variability by race and ethnicity," Prev. Med. (Baltim)., 2018.

37. Khan MMA, Karim M, Islam AZ, Islam MR, Khan HTA, Khalilullah MI. "Prevalence of overweight and obesity among adolescents in Bangladesh: Do eating habits and physical activity have a gender differential effect?," J. Biosoc. Sci., 2019.

\section{Tables}


Table-1Anthropometric and socio-demographic profile of adolescents aged 10-19 years in India, 201618

Variables

Body mass Index (BMI)

Not overweight/obese

Overweight/obese

Abdominalobesity

No

Yes

Triceps skinfold thickness (TSFT)

Not overweight

Overweight

Subscapular skinfold thickness (SSFT)

Not overweight

Overweight

Physical activity

Low

Medium

High

Serum triglycerides

Low

High

\section{Cholesterol}

Low

High

Low-density lipoprotein

Low

High

High-density lipoprotein
Male Female

Sample Percent Sample Percent

$\begin{array}{llll}15,697 & 95.0 & 14,841 & 95.1 \\ 821 & 5.0 & 761 & 4.9\end{array}$

\begin{tabular}{llll}
17,116 & 97.8 & 16,271 & 98.8 \\
\hline 381 & 2.2 & 202 & 1.2
\end{tabular}

16,675

94.7

16,276

98.3

937

5.3

276

1.7

16,356

92.9

15,826

95.7

1,249

7.1

704

4.3

4.3

6,963

37.8

12,178

70.0

4,975

27.0

2,544

14.6

6,486

35.2

2,683

15.4

\begin{tabular}{llll}
\hline 5,856 & 85.9 & 5,241 & 81.7 \\
\hline 963 & 14.1 & 1,172 & 18.3
\end{tabular}

$\begin{array}{llll}6,582 & 96.6 & 6,112 & 95.4 \\ 234 & 3.4 & 293 & 4.6\end{array}$

6,591

96.8

6,121

95.5

220

3.2

289

4.5 


\begin{tabular}{|c|c|c|c|c|}
\hline Low & 2,008 & 29.6 & 1,533 & 24.0 \\
\hline High & 4,780 & 70.4 & 4,854 & 76.0 \\
\hline \multicolumn{5}{|l|}{ Age (years) } \\
\hline Early adolescence (10-14) & 9,757 & 53.0 & 8,832 & 50.7 \\
\hline Late adolescence (15-19) & 8,668 & 47.0 & 8,573 & 49.3 \\
\hline \multicolumn{5}{|l|}{ Education } \\
\hline No schooling & 885 & 4.8 & 1,182 & 6.8 \\
\hline Dropout & 3,194 & 17.3 & 3,721 & 21.4 \\
\hline Currently studying & 14,346 & 77.9 & 12,502 & 71.8 \\
\hline \multicolumn{5}{|l|}{ Media exposure } \\
\hline No & 2,955 & 16.0 & 4,080 & 23.4 \\
\hline Yes & 15,470 & 84.0 & 13,325 & 76.6 \\
\hline \multicolumn{5}{|l|}{ Parent education } \\
\hline Both uneducated & 4,487 & 24.4 & 4,529 & 26.0 \\
\hline Anyone educated & 5,908 & 32.1 & 5,782 & 33.2 \\
\hline Both educated & 8,030 & 43.6 & 7,094 & 40.8 \\
\hline \multicolumn{5}{|l|}{ Parents diabetes status } \\
\hline No one & 17,675 & 95.9 & 16,769 & 96.4 \\
\hline Anyone had & 750 & 4.1 & 636 & 3.7 \\
\hline \multicolumn{5}{|l|}{ Diet } \\
\hline Vegetarian & 3,519 & 19.1 & 4,770 & 27.4 \\
\hline Non-vegetarian & 14,902 & 80.9 & 12,634 & 72.6 \\
\hline \multicolumn{5}{|l|}{ Junk food consumption } \\
\hline No & 4,876 & 26.5 & 4,638 & 26.7 \\
\hline 1-2 days & 12,771 & 69.3 & 12,216 & 70.2 \\
\hline 3-7 days & 774 & 4.2 & 549 & 3.2 \\
\hline \multicolumn{5}{|l|}{ Substance use } \\
\hline No & 16,876 & 91.6 & 17,194 & 98.8 \\
\hline Yes & 1,549 & 8.4 & 211 & 1.2 \\
\hline
\end{tabular}


Caste

$\mathrm{SC} / \mathrm{ST}$

5,769

33.2

5,769

33.2

Non-SC/ST

11,636

66.9

11,636

66.9

Religion

Hindu

14,878

80.8

13,797

79.3

Muslim

2,643

14.3

2,819

16.2

Christian

458

2.5

381

2.2

Others

446

2.4

408

2.3

Wealth quintile

\begin{tabular}{lllll} 
Poor & 7,292 & 39.6 & 7,036 & 40.4 \\
\hline Middle & 3,696 & 20.1 & 3,473 & 20.0 \\
\hline Rich & 7,437 & 40.4 & 6,896 & 39.6
\end{tabular}

\section{Place of residence}

\begin{tabular}{l} 
Rural \\
Urban \\
\hline Regions
\end{tabular}

North

13,788

74.8

13,174

75.7

Central

4,637

25.2

4,231

24.3

East

Northeast

2,597

14.1

2,306

13.3

West

South

Total

SC/ST: Scheduled caste/ Scheduled tribe 
Table-2Prevalence of pre-diabetes/diabetes among adolescents aged 10-19 years by sociodemographic characteristics in India, 2016-18

Variables

Male

Female(\%)

(\%)

Male-Female(\%) Proportion

Test $(p><.05)$

Body Mass Index (BMI)

Not overweight/obese

12.1

8.2

3.9

Overweight/obese

17.2

11.5

5.7

*

Abdominal obesity

$\begin{array}{lllll}\text { No } & 12.2 & 8.3 & 3.8\end{array}$ *

Triceps skinfold thickness (TSFT)

Not overweight

12.0

8.3

Overweight

18.6

10.5

8.0

Subscapular skinfold thickness (SSFT)

Not overweight

11.6

8.1

3.5

Overweight

22.6

12.8

9.8

Physical activity

Low

13.6

8.3

5.4

12.2

9.2

3.0

11.6

7.5

4.0

\section{Serum triglycerides}

Low

12.2

9.2

3.0

10.3

5.5

4.8

Cholesterol

Low

12.0

8.5

3.5

High

9.1

8.5

0.6

Low-density lipoprotein

Low

High
11.9

13.2
8.3

13.1

Page 16/19 
High-density lipoprotein

Low

High

Age (years)

Early adolescence (10-14)

Late adolescence (15-19)

\section{Education}

No schooling

Dropout

Currently studying

Media exposure

No

Yes

\section{Parent education}

Both uneducated

Anyone educated

Both educated

Parents diabetes status

No one

Anyone had

Diet

Vegetarian

Non-vegetarian

Junk food consumption

No

1-2 days

3-7 days

\section{Substance use}

No
8.7

12.3

13.0

11.7

8.7

7.8

8.2

7.7

0.5

12.3

9.4

8.1

12.6

11.8

7.1

4.7

12.6

8.6

4.0

4.6

4.0

12.5

8.0

4.5

12.7

9.3

3.4

12.3

7.7

4.6

12.5

8.4

4.2

10.2

6.7

3.5

0.0

14.1

10.3

12.0

7.6

4.5

3.9

10.7

2.2

4.7

12.1

7.5

5.7

8.5

14.1

5.7

*

11.9

8.2

3.7
$+$ 


\begin{tabular}{|c|c|c|c|c|}
\hline Yes & 18.9 & 14.1 & 4.8 & * \\
\hline \multicolumn{5}{|l|}{ Caste } \\
\hline SC/ST & 11.8 & 9.5 & 2.4 & * \\
\hline Non-SC/ST & 12.7 & 7.7 & 5.0 & * \\
\hline \multicolumn{5}{|l|}{ Religion } \\
\hline Hindu & 12.3 & 8.5 & 3.8 & * \\
\hline Muslim & 11.8 & 6.3 & 5.5 & * \\
\hline Christian & 18.1 & 12.3 & 5.8 & * \\
\hline Others & 15.3 & 10.1 & 5.1 & \\
\hline \multicolumn{5}{|c|}{ Wealth quintile } \\
\hline Poor & 10.8 & 9.4 & 1.4 & * \\
\hline Middle & 14.0 & 7.6 & 6.4 & * \\
\hline Rich & 13.3 & 7.6 & 5.6 & * \\
\hline \multicolumn{5}{|c|}{ Place of residence } \\
\hline Rural & 12.4 & 8.4 & 4.0 & * \\
\hline Urban & 12.7 & 8.0 & 4.7 & * \\
\hline \multicolumn{5}{|l|}{ Regions } \\
\hline North & 16.3 & 10.6 & 5.8 & * \\
\hline Central & 11.1 & 6.9 & 4.2 & * \\
\hline East & 9.3 & 5.5 & 3.7 & * \\
\hline Northeast & 18.4 & 8.3 & 10.1 & * \\
\hline West & 13.7 & 12.9 & 0.9 & \\
\hline South & 14.5 & 10.2 & 4.3 & * \\
\hline Total & 12.4 & 8.3 & 4.1 & * \\
\hline
\end{tabular}

*if p<0.05; SC/ST: Scheduled caste/ Scheduled tribe; \%: Percentage

Figures 


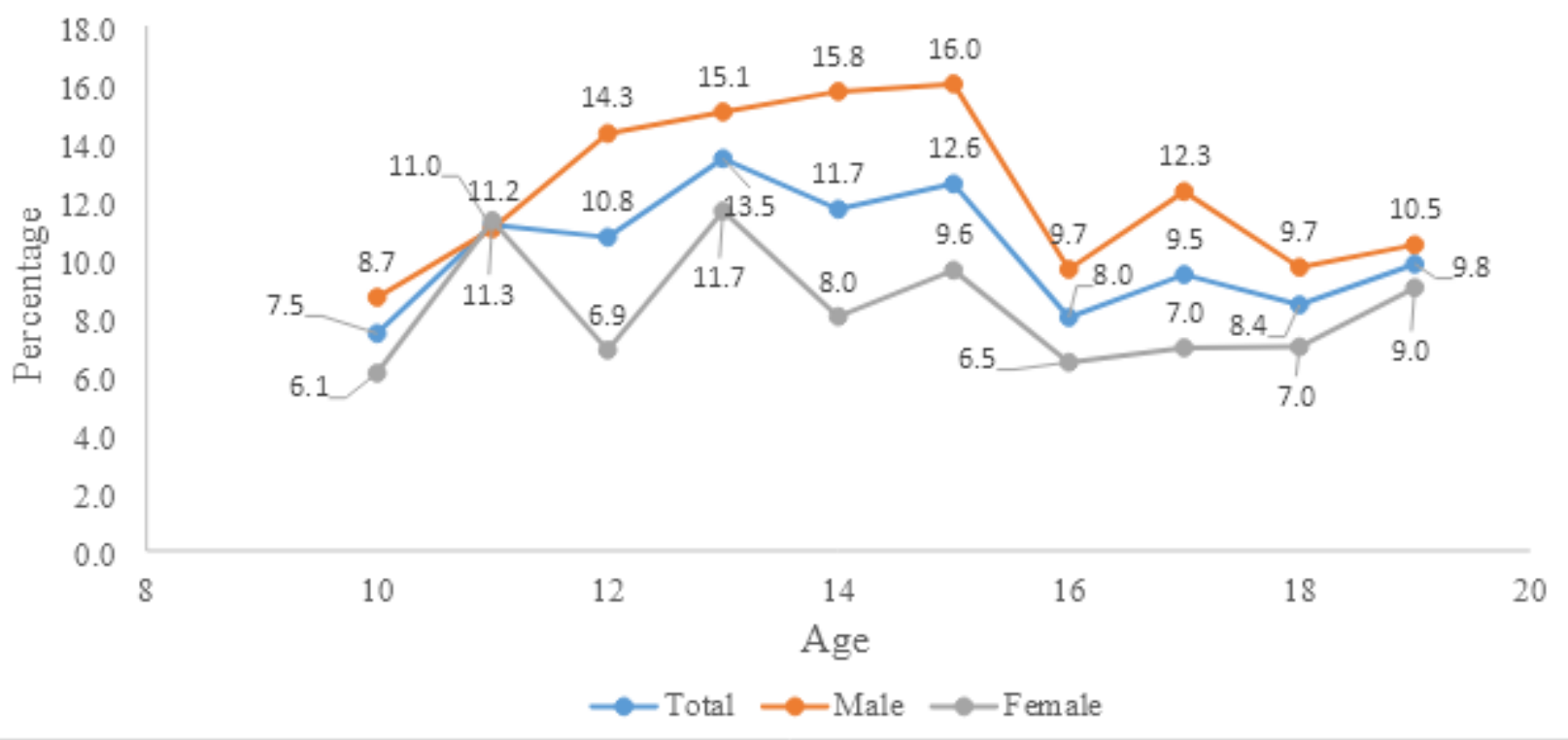

Figure 1

Gender differentials for pre-diabetes/diabetes among adolescents in India. 